Astron. Nachr. ... (...)

\title{
Internal shocks in blazar jets
}

\author{
GABRIELE GHiselLini \\ Osservatorio Astronomico di Brera, Via Bianchi 46, I-23807 Merate Italy
}

Received 1999 June 7; accepted ....

\begin{abstract}
The discovery of strong $\gamma$-ray and hard X-ray emission and the results from various multifrequency campaigns have disclosed new aspects of the blazar phenomenology, leading to a much more robust understanding of the mechanisms underlying their emission, and offering clues for the energetics of their relativistic jets. I review these aspects, and propose that all the diversity in the blazar phenomenology depends on only one parameter. I also suggest that some of the blazar characteristics can be explained by the internal shock scenario, as proposed to explain the emission from $\gamma$-ray bursts.
\end{abstract}

Key words: blazars; radiation processes, jets, $\gamma$-rays.

\section{Introduction}

Fossati et al. (1998) and Ghisellini et al. (1998) proposed that the blazar diversity can be framed in a coherent picture, where blazars form a sequence according to their observed bolometric luminosity. All blazars have a spectral energy distribution (SED) with two peaks in a $\nu-\nu F_{\nu}$ plot: we believe that the low frequency peak (between the far IR and $\mathrm{X}$-ray band) is due to synchrotron emission, and the high energy peak (between the MeV and $\mathrm{TeV}$ bands) to inverse Compton emission produced by the same electrons. As the luminosity increases, both peaks shift to lower frequencies, and the inverse Compton power increases its dominance (i.e. the ratio between the inverse Compton $\gamma$-ray luminosity and the synchrotron power, $L_{c} / L_{s}$ ). By fitting the SED of blazars with a synchrotron-self Compton model which includes the possible contribution of radiation produced outside the jet (namely radiation directly from the broad line region, or scattered by intercloud material, or from the walls of the jet), we can estimate the physical quantities inside the emitting region, such as the magnetic field, the emitting particle density and the shape of their energy distribution, together with the Lorentz factor of the bulk motion of the region and its dimension. Even if the results may be affected by large uncertainties in single sources, due mainly to the paucity of simultaneous spectra, they are meaningful when derived for a relatively large sample of sources in a statistical sense. One of the most important parameters is the random Lorentz factor, $\gamma_{p e a k}$, of the particles emitting most of the radiation, i.e. the ones responsible for the emission at both peaks of the SED. We find that $\gamma_{\text {peak }}$ strongly correlates with the intrinsic energy density $U$ (both radiative and magnetic), the total intrinsic power, and the amount of Compton dominance. This both unifies and explains the different sub-classes of blazars: low luminosity objects are characterized by small energy densities and large values of $\gamma_{\text {peak }}$, producing a SED peaking at high energies, as the one typical of "high frequency peaked" BL Lac objects (Giommi \& Padovani 1994). External radiation (e.g. emission lines) is absent in these sources, and this results into $L_{c} / L_{s}$ of order unity or less. At the other extreme, powerful blazars with strong emission lines are characterized by large values of $U$ and small $\gamma_{\text {peak }}$ : their SED peaks at low energies, and the presence of external radiation boosts the inverse Compton process, producing highly Compton dominated sources.

But where and how is this radiated energy produced? It is widely believed that the radiation in the jet comes from the transformation of kinetic energy of the bulk motion into random energy. Here I will suggest that several characteristic of blazars, including those just described, can be explained by an internal shock scenario, where shells of slightly different velocities interact at some distance from the central engine.

\section{Internal shocks}

Rees (1978) faced the problem of explaining the optical emission of the jet of M87. The large energies of the emitting electrons imply very short cooling times, excluding the possibility of transporting those electrons from the nucleus. In situ acceleration is necessary. A way to achieve this is to envisage a central engine working not continuously, but producing different shells of material moving with different bulk Lorentz factors $\Gamma$. A faster shell 
$2 \Gamma$ and $R_{0}$ is the initial separation of the two shells. Curiously, this idea was more or less forgotten in the blazar field (but see Sikora, Begelman \& Rees 1994), but became the standard scenario to explain the emission of $\gamma$-ray bursts (GRB; Rees \& Meszaros 1994).

Note that internal shocks are different from the so called external shock scenario - proposed to explain the afterglow emission of GRBs, and recently considered by Dermer (1999) to also explain the blazar emission where the $\gamma$-ray fireball/'blob' in blazars decelerates interacting with the interstellar medium. I will present in the following some arguments in favor of the internal shock scenario operating in the blazar jet.

\subsection{Shock location}

We know, mainly thanks to EGRET onboard CGRO, that most of the emission of blazars is in the $\gamma$-ray band. This emission, and the fact that it varies with short timescales, implies that there must be a preferred location where dissipation of the bulk motion energy occurs. On one hand, if it were at the base of the jet, and hence close to the accretion disk, the produced $\gamma$-rays should be inevitably absorbed by photon-photon collisions, with associated copious pair production, reprocessing the original power from the $\gamma$-ray to the $\mathrm{X}$-ray part of the spectrum (contrary to observations). On the other, if it were far away, in a large region of the jet, it becomes difficult to explain the observed fast variability, even accounting for the time-shortening due to the Doppler effect. Regions distant $\sim 10^{17}$ $\mathrm{cm}$ from the base of the jet are then selected. This distance is likely to be within the broad line region: as the extra seed photons provided by emission lines enhance the efficiency of the Compton process responsible for the $\gamma$-ray emission. And indeed this is the typical distance at which two shells, initially separated by $R_{0} \sim 10^{15} \mathrm{~cm}$ (comparable to a few Schwarzschild radii), moving with $\Gamma \sim 10$ would collide.

\subsection{Time variability}

The light curve of GRBs is extremely variable and spiky, with the shortest spikes lasting for $1 \mathrm{~ms}$. Suppose that a single spike corresponds to a single shell-shell encounter, and that its duration is determined by the time needed to the two shells to cross each other. If the shell widths is of the same order of their separation, this is given by the time elapsed to go from $R_{i}$ to $2 R_{i}$. But the observer will see the crossing time Doppler contracted by the factor $(1-\beta \cos \theta) \sim \Gamma^{-2}$ (where $\theta \sim 0^{\circ}$ is the viewing angle), since the shell is moving almost at the speed of light. The shortest spikes of the GRB light curves can be therefore associated to the size of the central engine (i.e. $R_{0}$ ), since $t_{\text {obs }} \sim R_{i} /\left(c \Gamma^{2}\right) \sim R_{0} / c$.

If the mechanism powering GRB and blazar emission is the same, we should expect a similar light curve from both systems, but with times appropriately scaled by the different $R_{0}$, i.e. the different masses of the involved black holes. Indeed, if one stretches a GRB light curve by a factor $\sim 10^{8}$, one obtains light curves very similar to the blazar ones, with the shortest timescales of the order of hours-days. In addition, by examining GRB light curves through Fourier analysis and adding up the resulting power spectra, a remarkably well defined power law (in frequency space) is found, with slope $-5 / 3$, characterizing a Kolmogoroff spectrum (Belobodorov, Stern \& Svensson 1998). This may be also the case for blazars.

\subsection{Efficiency}

Most of the power transported by the jet must reach the radio lobes. Hence only a small fraction can be radiatively dissipated. The efficiency $\eta$ of two blobs/shells for converting ordered into random energy depends on their masses $m_{1}, m_{2}$ and bulk Lorentz factors $\Gamma_{1}, \Gamma_{2}$, as

$$
\eta=1-\Gamma_{f} \frac{m_{1}+m_{2}}{\Gamma_{1} m_{1}+\Gamma_{2} m_{2}}
$$

where $\Gamma_{f}=\left(1-\beta_{f}^{2}\right)^{-1 / 2}$ is the bulk Lorentz factor after the interaction and is given by (see e.g. Lazzati, Ghisellini \& Celotti 1999)

$$
\beta_{f}=\frac{\beta_{1} \Gamma_{1} m_{1}+\beta_{2} \Gamma_{2} m_{2}}{\Gamma_{1} m_{1}+\Gamma_{2} m_{2}}
$$

The above relations imply, for shells of equal masses and $\Gamma_{2}=2 \Gamma_{1}=20, \Gamma_{f}=14.15$ and $\eta=5.7 \%$.

Efficiencies $\eta$ around $5-10 \%$ are just what needed for blazar jets. Furthermore, we can "directly" estimate the values appropriate for blazars from the amounts of power radiated and transported by jets in the form of cold protons, magnetic field and hot electrons and/or electron-positron pairs. This requires to determine the bulk 


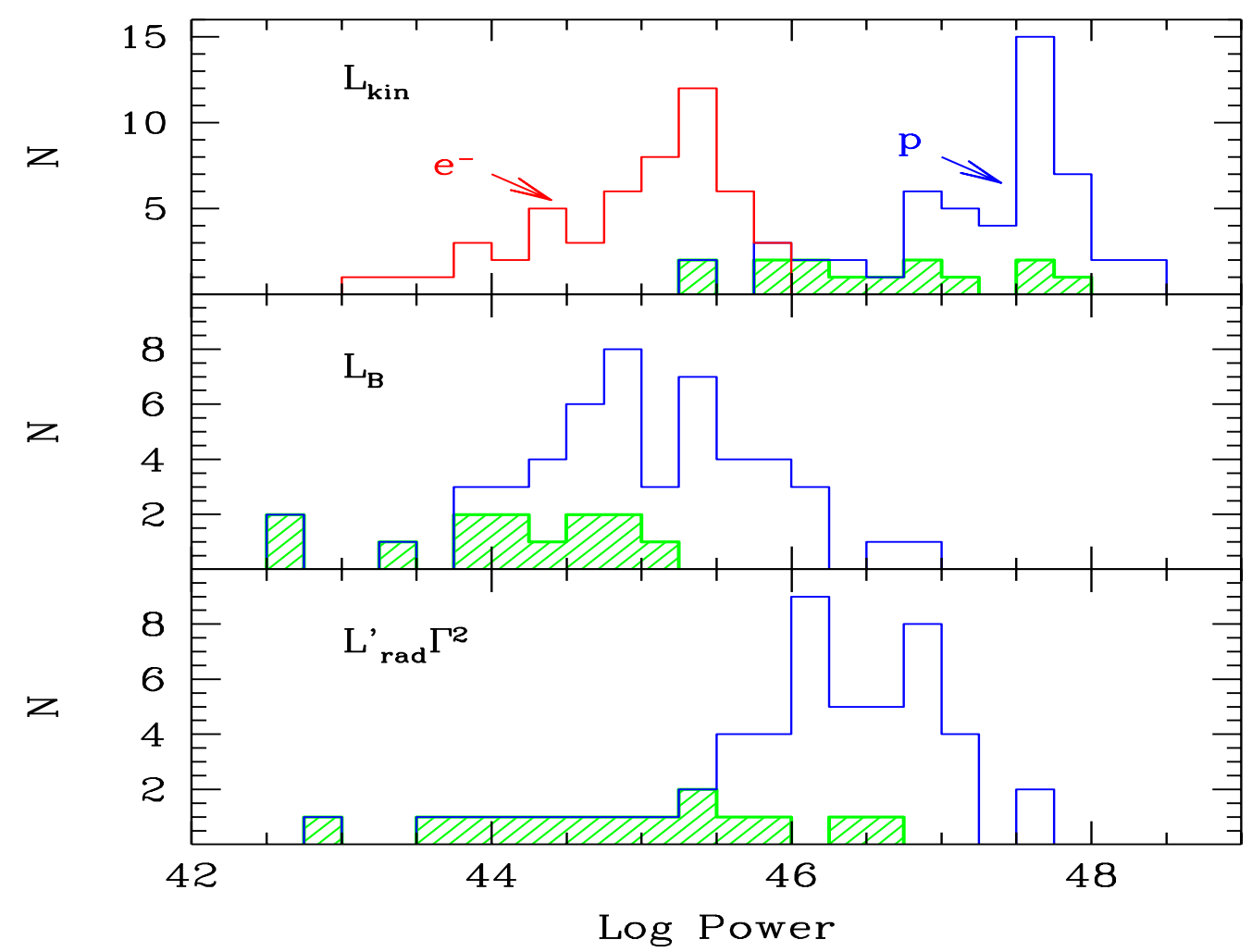

Fig. 1: Histograms of the power transported in the form of cold protons and relativistic electrons (upper panel), Poynting vector (middle panel) and the emitted luminosity (bottom panel). Shaded areas correspond to BL Lac objects in all histograms but $L_{e}$ (in this case the shaded area has been omitted for clarity). From Celotti \& Ghisellini 1999, in prep.

Ghisellini et al. (1998) have computed these quantities for all (51) blazars detected by EGRET for which both the redshift and the $\gamma$-ray spectral shape are currently known, and thus determined

$$
L_{p}=\pi R^{2} \Gamma^{2} \beta c n_{p}^{\prime} m_{p} c^{2} ; \quad L_{e}=\pi R^{2} \Gamma^{2} \beta c n_{e}^{\prime}\langle\gamma\rangle m_{e} c^{2} ; \quad L_{B}=\pi R^{2} \Gamma^{2} \beta c \frac{B^{2}}{8 \pi}
$$

These powers can be compared with the radiated one estimated in the same frame (in which the emitting blob is seen moving). The power radiated in the entire solid angle is thus $L=L^{\prime} \Gamma^{2}$. All these quantities are plotted in Fig. 1 (Celotti \& Ghisellini 1999, in prep.), from which it can be noted that:

- If the jet is formed by electrons-positrons only, the corresponding kinetic power is smaller than the radiated power, posing a serious energy budget problem.

- If there is a proton for each electron, the bulk kinetic power is $\sim 10$ times larger than the radiated one. This value agrees with the energy requirements of the radio lobes.

- The power in the Poynting flux is of the same order of $L_{e}$, indicating that the magnetic field is close to the equipartition with the electron energy density. This suggests that the magnetic field is not a prime energy carrier, but that is a sub-product of the interaction transforming bulk into random energy.

- The power in electrons is smaller than the radiated power, by a factor $\sim 10$. This requires reacceleration/continuous injection of electrons.

\subsection{Spectrum}

The internal shock scenario allows to estimate the total energy available for radiative dissipation. In fact, in the rest frame of the fast shell, the bulk kinetic energy of each proton of the slower shell is $\sim\left(\Gamma^{\prime}-1\right) m_{p} c^{2}$, where $\Gamma^{\prime} \sim 2$. This is what can be transformed into random energy. Assume now that the electrons share some of this available energy (through an unspecified acceleration mechanism). In the comoving frame, the acceleration rate 


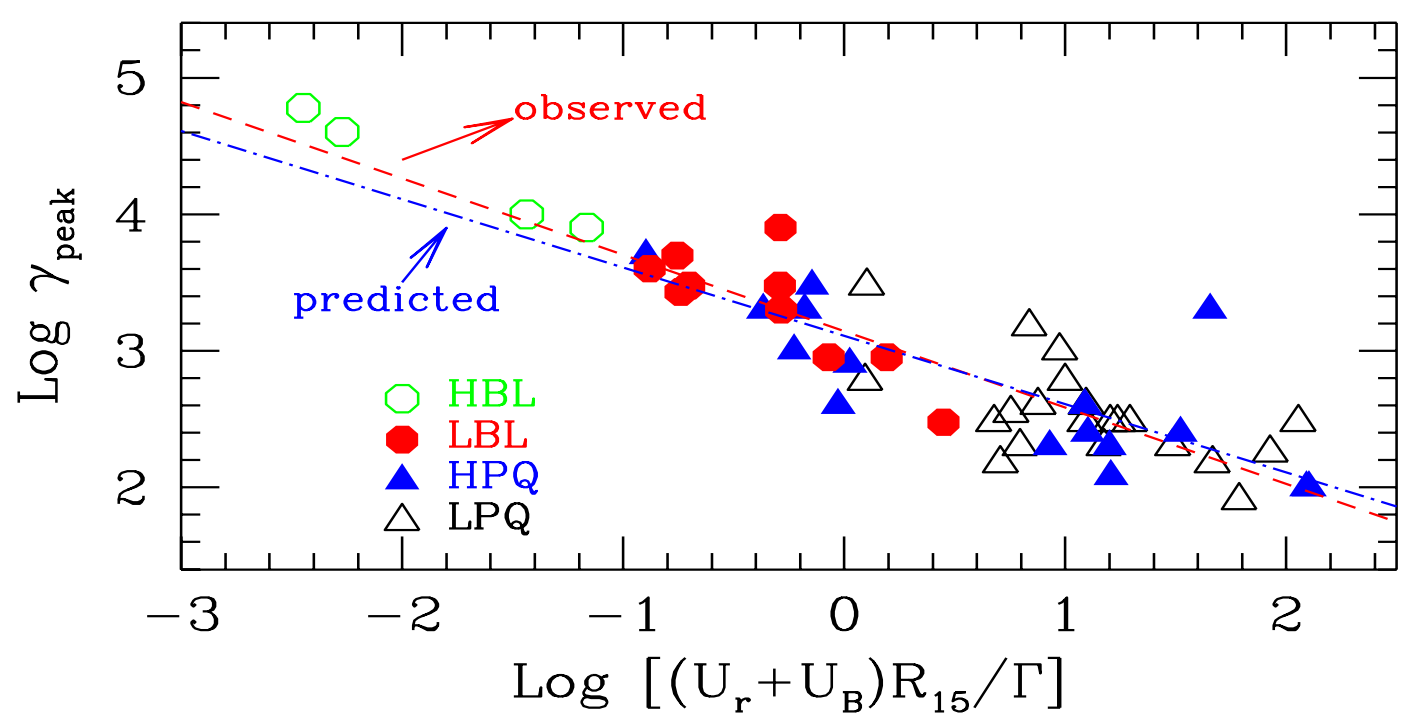

Fig. 2: Correlation between the Lorentz factor of the electrons emitting at the peaks of the SED (both synchrotron and inverse Compton) and the quantity $U R / \Gamma$, where $U=U_{r}+U_{B}$ is the sum of radiation and magnetic energy densities, $R / \Gamma$ is the shell width $\left(R=10^{15} R_{15} \mathrm{~cm}\right)$. Labels indicate High and Low frequency peak BL Lac objects (HBL and LBL), and High and Low polarization quasars (HPQ and LPQ, respectively).

the two shells to cross, i.e. $t_{\text {heat }}^{\prime} \sim \Delta R^{\prime} / c \sim R /(c \Gamma)$, where $\Delta R^{\prime}$ is the shell width (measured in the same frame). The heating and the radiative cooling rates will balance for some value of the random electron Lorentz factor $\gamma_{\text {peak }}$ :

$$
\dot{E}_{\text {heat }}=\dot{E}_{\text {cool }} \rightarrow \frac{\Gamma m_{p} c^{3}}{R}=\frac{4}{3} \sigma_{T} c U \gamma_{\text {peak }}^{2} \rightarrow \gamma_{\text {peak }}=\left(\frac{3 \Gamma m_{p} c^{2}}{4 \sigma_{T} R U}\right)^{1 / 2}
$$

This prediction can be tested against the parameters derived by the fitting of the EGRET blazars, described above, and the result is shown in Fig. 2. The agreement is surprisingly good both in the relative dependence and in the absolute normalization.

\section{Conclusions}

The idea that blazar emission can originate from internal shocks seems promising. In addition, the similarity with GRBs phenomenology suggests that internal shocks might be one chief mechanism to convert relativistic bulk motion into radiation.

Acknowledgements. It is a pleasure to thank Annalisa Celotti, for the ongoing collaboration within which the ideas reported in this work have been conceived.

\section{References}

Belobodorov, A.M., Stern, B. \& Svensson, R.: 1998, ApJ, 508, L25

Dermer, C.D.: 1999, Astroparticle Physics, ed. M. Catanese, J. Quinn, T. Weekes, in press astro-ph/9901324

Fossati, G., Maraschi, L. Celotti, A., Comastri, A. \& Ghisellini G.: 1998, MNRAS, 299, 433

Ghisellini, G., Celotti, A., Fossati, G., Maraschi, L. \& Comastri, A.: 1998, MNRAS, 301, 451

Lazzati, D., Ghisellini, G. \& Celotti, A.: 1999, submitted to MNRAS

Giommi,P. \& Padovani, P.: 1994, ApJ, 444, 567

Rees, M.J.: 1978, MNRAS, 184, P61

Rees, M.J. \& Meszaros P.: 1994, ApJ, 430, L93

Sikora, M., Begelman, M.C. \& Rees, M.J.: 1994, ApJ, 421, 153 